\title{
Hiperparatiroidismo durante el embarazo. ¿Es un reto diagnóstico y terapéutico?
}

\author{
Hyperparathyroidism during pregnancy. Is it a diagnostic and therapeutic challenge?
}

Ansony R. Godínez-Vidal*, Édgar Montes de Oca-Durán, Vania I. Aguirre-Rojano, Abraham Pulido-Cejudo, Erich O. Paul-Basurto-Kuba y Luis M. Hurtado-López

Departamento de Cirugía General, Hospital General de México “Dr. Eduardo Liceaga”, Ciudad de México, México

\begin{abstract}
Resumen
Introducción: El hiperparatiroidismo primario (HPTP) se caracteriza por un aumento de la hormona paratiroidea (PTH) e hipercalcemia, que aumenta la morbimortalidad materna y fetal cuando se presenta durante el embarazo. Objetivo: Enfatizar la importancia de la intervención quirúrgica en el hiperparatiroidismo primario durante el embarazo. Caso clínico: Paciente femenino de 27 años de edad con embarazo de 27.2 semanas de gestación, con diagnóstico de hiperparatiroidismo primario sintomático secundario a adenoma paratiroideo, antecedentes de nefrolitiasis y pancreatitis aguda grave; al decidir realizar un procedimiento quirúrgico se identificó un adenoma paratiroideo derecho intratiroideo; el curso posquirúrgico mostró adecuada evolución y remisión del hiperparatiroidismo. Conclusión: La paratiroidectomía en el hiperparatiroidismo primario durante el embarazo es segura.
\end{abstract}

PALABRAS CLAVE: Hiperparatiroidismo primario. Hormona paratiroidea. Adenoma paratiroideo. Paratiroidectomía. Embarazo.

\begin{abstract}
Background: Primary hyperparathyroidism (PHPT) is characterized by an increase in parathyroid hormone (PTH) and hypercalcemia, which, when present during pregnancy, increases both maternal and fetal morbidity and mortality. Objective: Emphasize the importance of surgical intervention in primary hyperparathyroidism during pregnancy. Clinical case: A 27-year-old female with a pregnancy of 27.2 weeks of gestation, with a diagnosis of symptomatic primary hyperparathyroidism secondary to parathyroid adenoma, a history of nephrolithiasis and severe acute pancreatitis, surgery was decided upon finding intrathyroid right parathyroid adenoma, post-surgical course with adequate evolution and remission of hyperparathyroidism. Conclusions: Parathyroidectomy in primary hyperparathyroidism during pregnancy is safe.
\end{abstract}

KEY WORDS: Primary hyperparathyroidism. Parathyroid hormone. Parathyroid adenoma. Parathyroidectomy. Pregnancy.

\section{Introducción}

Las paratiroides son glándulas pequeñas, casi siempre situadas en el cuello en la cara posterolateral de la glándula tiroides. Su función principal es producir la hormona paratiroidea (PTH) que interviene en el metabolismo del calcio y fósforo. Cuando la paratiroides, sin ningún estímulo secundario, aumenta su función endocrina y produce exceso de hormona paratiroidea se presenta el hiperparatiroidismo primario (HPTP) ${ }^{1}$.

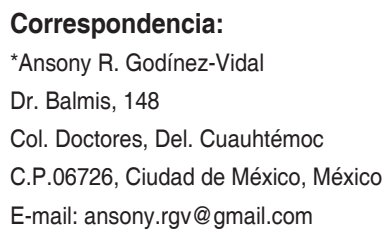

Cir Cir. 2019;87(S1):62-67

Contents available at PubMed www.cirugiaycirujanos.com 
El HPTP provoca hipercalcemia y ello causa en distinto grado afectación de diversos órganos y sistemas, que acentúa la morbimortalidad materna y fetal cuando se presenta en el embarazo. El HPTP en la gestación es una enfermedad que presenta particularidades desde los puntos de vista fisiológico, pronóstico y terapéutico, lo que conlleva una mayor dificultad diagnóstica y un elevado riesgo de complicaciones. Es la tercera causa de enfermedad endocrina en la mujer embarazada, después de la diabetes y la enfermedad tiroidea, y es la causa más común de hipercalcemia ${ }^{2,3}$.

La prevalencia del HPTP detectado durante el embarazo es de $0.15 \%$ a $1.4 \%$. A menudo no se diagnostica debido a una presentación asintomática e inespecífica en el $80 \%$ de los casos debido a que los cambios fisiológicos del embarazo atenúan los signos clínicos ${ }^{4}$. Su incidencia en mujeres en edad fecunda es de 8/100 000 pacientes por año $0^{5}$. Algunas alteraciones fisiológicas propias de la mujer gestante contribuyen a ocultar la hipercalcemia, entre ellas el aumento de la tasa de filtración glomerular y la excreción urinaria de calcio, el incremento del volumen del líquido extracelular, la hipoalbuminemia y la gran demanda fetal de calcio. Si la gestante desarrolla hipercalcemia se puede producir una supresión de las paratiroides fetales, con el consiguiente riesgo de tetania neonatal ${ }^{6}$. Más del $80 \%$ de los HPTP en gestantes cursan de forma asintomática ${ }^{7}$, lo que dificulta la detección de la hipercalcemia y hace que sea un proceso subdiagnosticado en el embarazo y que se desconozca su incidencia real. Desde el primer caso de HPTP en el embarazo que notificaron en 1931 Hunter y Turn-Bull ${ }^{8}$, alrededor de 200 casos se han publicado en la bibliografía médica. En las gestantes, la causa más frecuente es el adenoma paratiroideo único, que causa 80 a $85 \%$ de los casos. El 15 a $20 \%$ restante puede deberse a hiperplasias difusas y con menos frecuencia a adenomas dobles o triples (2\%) o carcinomas $(<1 \%)^{9}$.

La presentación del cuadro incluye hiperemesis, nefrolitiasis, infección urinaria recurrente y pancreatitis. La nefrolitiasis es la complicación más frecuente, con una incidencia del 24 al $36 \%$. En estas pacientes existe, además, un incremento significativo de la incidencia de hiperemesis gravídica, arritmias cardiacas, abortos espontáneos, síndrome de preeclampsia-eclampsia, parto prematuro, hipertensión arterial, infecciones del tracto urinario y pielonefritis. El riesgo de presentar un episodio de pancreatitis aguda también se encuentra incrementado en las mujeres gestantes $(7-13 \%)$ con respecto a las no gestantes con HPTP $(1-2 \%)^{6,10}$. Las complicaciones neonatales incluyen hipocalcemia y tetania, secundarias a la supresión de la hormona paratiroidea fetal, polihidramnios, bajo peso al nacer y defunción fetal. Cuando se interpreta la gravedad de las elevaciones séricas de calcio en mujeres embarazadas, es importante recordar que el calcio sérico total decrece a lo largo de la gestación, probablemente debido a la expansión del volumen plasmático. El límite superior de la normalidad para el calcio sérico total es de $9.5 \mathrm{mg} / \mathrm{dl}$ en el embarazo ${ }^{11-14}$.

\section{Objetivo}

Presentar este informe de caso para enfatizar la importancia de la adecuada intervención médica y quirúrgica en el hiperparatiroidismo primario durante el embarazo.

\section{Caso clínico}

Se trata de un paciente femenino de 27 años de edad con diagnóstico de embarazo de 27.2 semanas de gestación e hiperparatiroidismo primario secundario a adenoma paratiroideo; cuenta con antecedente de tres embarazos, dos abortos (uno inducido y otro por muerte fetal en el segundo trimestre del embarazo) y nefrolitiasis. Dos meses antes de su ingreso presentó pancreatitis aguda grave secundaria a hipercalcemia, la cual requirió atención medica en terapia intensiva de otro hospital durante 15 días y necesidad de transfusión de hemocomponentes. Ingresó a la institución de los autores por dolor abdominal de predominio en hipogastrio, náusea sin vómito, y ningún otro síntoma. A la exploración física se palpa fondo uterino congruente con 27 semanas de gestación y contracciones que cedieron con la hidratación. Debido a los antecedentes de nefrolitiasis, pancreatitis e hipercalcemia se sospechó hiperparatiroidismo que se corroboró con los estudios de laboratorio (PTH, $341.5 \mathrm{pg} / \mathrm{ml}$ ) (Tabla 1). El ultrasonido de cuello mostró imagen nodular de 16 × 6.1 × $13 \mathrm{~mm}$, volumen aproximado de $0.65 \mathrm{~cm}^{3}$ de bordes regulares y márgenes bien circunscritos (Fig. 1). Se decide realizar una intervención en la que se encuentra adenoma paratiroideo inferior derecho intratiroideo (Fig. 2), por lo que se practica hemitiroidectomía derecha, sin presentarse complicaciones durante la operación y curso posquirúrgico de adecuada evolución. Se realizan pruebas de laboratorio tras la intervención, sin 
Tabla 1. Estudios de laboratorios antes de la operación

\begin{tabular}{|c|c|c|}
\hline Hormona & Cifras séricas & Cifras normales \\
\hline Hormona paratiroidea (PTH) & $341.5 \mathrm{pg} / \mathrm{ml}$ & $12-88 \mathrm{pg} / \mathrm{ml}$ \\
\hline Hormona estimulante de la tiroides (TSH) & $2.03 \mathrm{mUl} / \mathrm{ml}$ & $\begin{array}{c}\text { (1er trimestre: } 0.05-3.70 \mathrm{mUl} / \mathrm{ml}) \\
\text { (2do trimestre: } 0.31-4.35 \mathrm{mUl} / \mathrm{ml} \text { ) } \\
\quad(3 e r \text { trimestre: } 0.41-5.18)\end{array}$ \\
\hline Triyodotironina libre (T3L) & $3.10 \mathrm{mUl} / \mathrm{ml}$ & $2.39-6.79 \mathrm{mUl} / \mathrm{ml}$ \\
\hline Tiroxina libre (T4L) & $0.47 \mathrm{mUl} / \mathrm{ml}$ & $0.58-1.64 \mathrm{mUl} / \mathrm{ml}$ \\
\hline Hormona foliculoestimulante (FSH) & $4.85 \mathrm{mUl} / \mathrm{ml}$ & $\begin{array}{c}\text { Fase folicular: } 3.85-8.78 \mathrm{mUI} / \mathrm{MI} \text { ) } \\
\text { (fase ovulatoria, } 4.54-22.51 \mathrm{mUI} / \mathrm{mL} \text { ) } \\
\text { (fase lútea: } 1.79-5.12 \mathrm{mUI} / \mathrm{mL} \text { ) } \\
\text { (posmenopausia: } 16.74-113.59 \mathrm{mUl} / \mathrm{m} \text { ) }\end{array}$ \\
\hline Cortisol & $10.05 \mathrm{mUl} / \mathrm{ml}$ & (matutina: 6.7-22.6 mUl/ml) (vespertina: $<10.0 \mathrm{mUl} / \mathrm{ml}$ ) \\
\hline Fósforo & $2.1 \mathrm{mg} / \mathrm{dl}$ & 2.4 a $4.7 \mathrm{mg} / \mathrm{dl}$ \\
\hline Calcio & $14.5 \mathrm{mg} / \mathrm{dl}$ & 8.5 a $10.5 \mathrm{mg} / \mathrm{dl}$ \\
\hline Creatinina & $1.5 \mathrm{mg} / \mathrm{dl}$ & 0.8 a $1.4 \mathrm{mg} / \mathrm{dl}$ \\
\hline
\end{tabular}

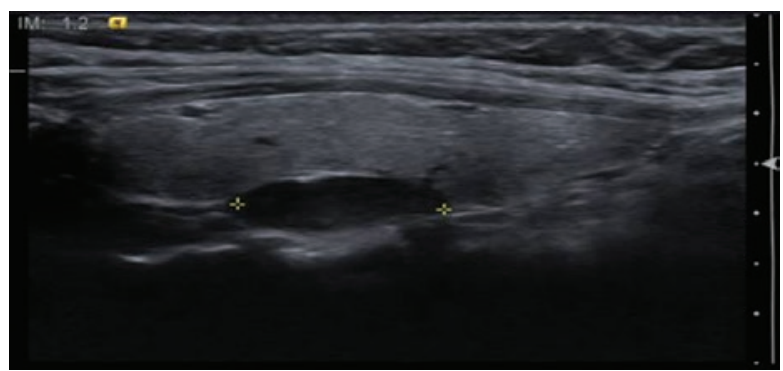

Figura 1. En el ultrasonido se observa nodulación hipoecoica de $16 x$ $6.1 \times 13 \mathrm{~mm}$ posterior al lóbulo derecho de la glándula tiroides.

encontrar alteraciones (Tabla 2), por lo que egresa una semana después del acto quirúrgico. El estudio histopatológico notificó adenoma paratiroideo y microcarcinoma papilar de tiroides (Fig. 3). Con posterioridad acudió a la institución al término del embarazo donde se realizó cesárea, sin complicaciones, y se obtuvo producto eutrófico que no presentó datos de hipocalcemia y la evolución fue favorable. En relación con el microcarcinoma papilar de tiroides, la paciente quedó en observación ya que se trata de un tumor de bajo riesgo.

\section{Calcio durante el embarazo}

La homeostasis del calcio en el embarazo está adaptada para promover el aporte activo de 25 a $30 \mathrm{~g}$ de calcio al feto y conservar la estructura ósea materna. Estos mecanismos de regulación se controlan sobre todo por acción del calcio no receptor específico y por varias hormonas, la principal de ellas la
PTH. El papel de la PTH en el embarazo no está bien definido, pero sus acciones son múltiples, como participar en la homeostasis del calcio, la embriogénesis, la formación del esqueleto fetal y la regulación del calcio materno $0^{6,10}$.

El exceso de PTH actúa sobre el hueso, con aumento de la función osteoclástica y provoca así un incremento del calcio sérico. En el riñón aumenta la eliminación de fósforo y la reabsorción de calcio. Se eleva, además, la vitamina D activada, que en el intestino también acentúa la reabsorción de calcio. Todo esto hace que el exceso de PTH eleve el calcio en sangre y reduzca la cantidad de fósforo. Durante el embarazo se produce una transferencia de calcio y fósforo maternos al feto. El paso excesivo de calcio al feto provoca la supresión de la secreción de PTH fetal y el incremento de los valores de calcitonina para favorecer, de esta manera, la mineralización ósea fetal. Esto se vincula con una alta morbimortalidad maternofetal ${ }^{15}$. Tras el parto, el neonato se encuentra súbitamente privado del aporte incrementado de calcio materno; además, es incapaz de movilizar sus reservas óseas por los reducidos valores de PTH y la elevada concentración de calcitonina. Como consecuencia de ello, entre los días 5 y $14^{\circ}$ pueden presentarse episodios de hipocalcemia neonatal con tetania, e incluso convulsiones si no se corrige de forma temprana. Otras complicaciones fetales incluyen el retraso del crecimiento intrauterino, el parto de pretérmino, el aborto y la muerte neonatal; estas complicaciones son más frecuentes 


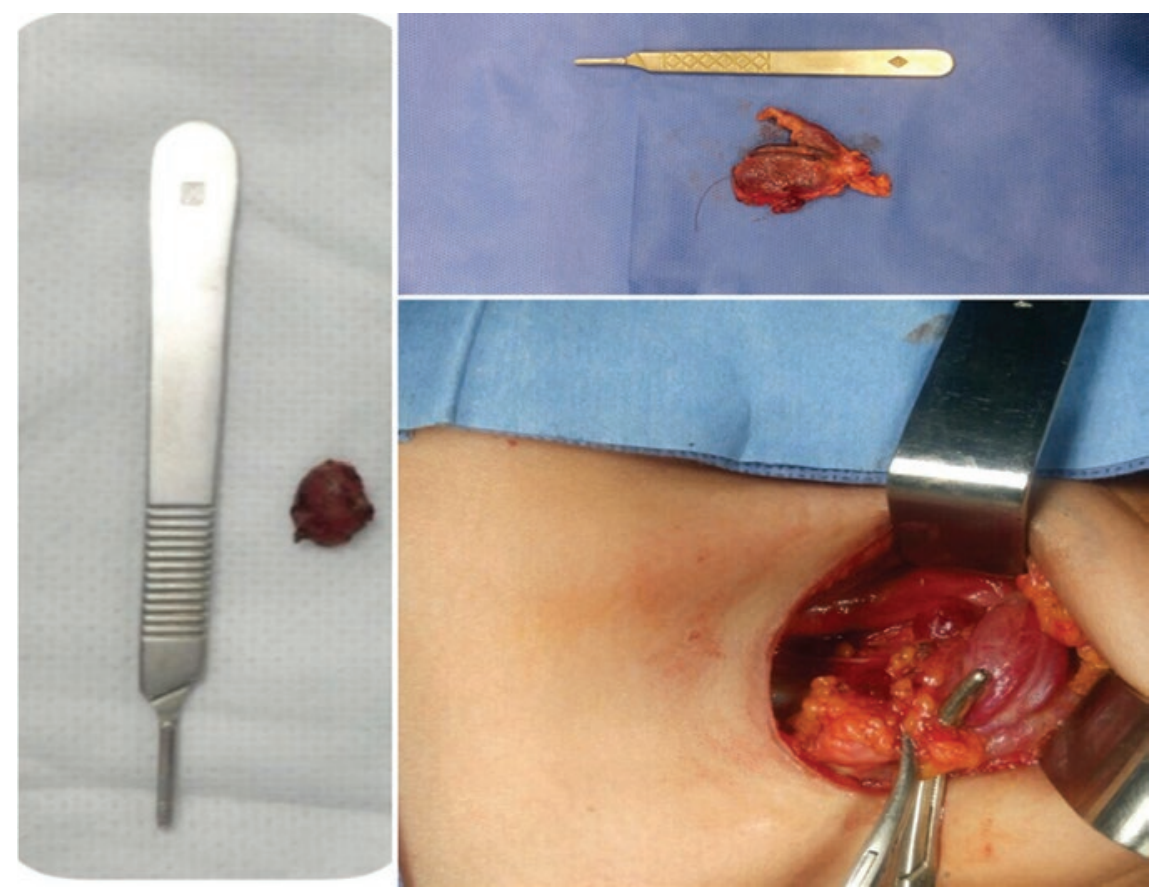

Figura 2. Adenoma de la paratiroides inferior derecha dentro de la glándula tiroides.

Tabla 2. Estudios de laboratorios en el postoperatorio inmediato

\begin{tabular}{|c|c|c|}
\hline Hormona & Valores séricos & Valores normales \\
\hline Hormona paratiroidea (PTH) & $75 \mathrm{pg} / \mathrm{ml}$ & $12-88 \mathrm{pg} / \mathrm{ml}$ \\
\hline Hormona estimulante de la tiroides (TSH) & $5.00 \mathrm{mUl} / \mathrm{ml}$ & $\begin{array}{c}\text { (1er trimestre: } 0.05-3.70 \mathrm{mUl} / \mathrm{ml} \text { ) } \\
\text { (2do trimestre: } 0.31-4.35 \mathrm{mUl} / \mathrm{ml} \text { ) } \\
\quad \text { (3er trimestre: } 0.41-5.18 \text { ) }\end{array}$ \\
\hline Triyodotironina libre (T3L) & $3.59 \mathrm{mUI} / \mathrm{ml}$ & $2.39-6.79 \mathrm{mUI} / \mathrm{ml}$ \\
\hline Tiroxina libre (T4L) & $0.75 \mathrm{mUl} / \mathrm{ml}$ & $0.58-1.64 \mathrm{mUI} / \mathrm{ml}$ \\
\hline Hormona foliculoestimulante (FSH) & $3.95 \mathrm{mUl} / \mathrm{ml}$ & $\begin{array}{l}\text { Fase folicular: 3.85-8.78 mUI/MI) } \\
\text { (fase ovulatoria: } 4.54-22.51 \mathrm{mUl} / \mathrm{mL} \text { ) } \\
\text { (fase lútea: } 1.79-5.12 \mathrm{mUI} / \mathrm{mL} \text { ) } \\
\text { (posmenopausia: } 16.74-113.59 \mathrm{mUl} / \mathrm{m} \text { ) }\end{array}$ \\
\hline Cortisol & $12.05 \mathrm{mUl} / \mathrm{ml}$ & (matutina: 6.7-22.6 mUl/ml) (vespertina: $<10.0 \mathrm{mUl} / \mathrm{ml}$ ) \\
\hline Fósforo & $3.2 \mathrm{mg} / \mathrm{dl}$ & 2.4 a $4.7 \mathrm{mg} / \mathrm{dl}$ \\
\hline Calcio & $9.1 \mathrm{mg} / \mathrm{dl}$ & 8.5 a $10.5 \mathrm{mg} / \mathrm{dl}$ \\
\hline Creatinina & $1 \mathrm{mg} / \mathrm{dl}$ & 0.8 a $1.4 \mathrm{mg} / \mathrm{dl}$ \\
\hline
\end{tabular}

en los casos de madres con HPTP que se trataron de forma conservadora ( $37 \%$ con complicaciones y $16 \%$ con muertes neonatales) que en las sometidas a operación ( $10 \%$ con complicaciones y $2.5 \%$ con muertes neonatales $)^{16}$.

Durante el embarazo se produce un descenso de los valores de PTH. Davison, et al. ${ }^{17}$ midieron la PTH de 11 embarazadas y 81 mujeres no embarazadas, y detectaron valores más altos en el $72 \%$ de las no embarazadas. Los valores medios se sitúan en el límite inferior de la normalidad en el primer trimestre y alcanzan valores medios de la normalidad al final de la gestación ${ }^{18}$. Este cambio es secundario a la modificación de las concentraciones plasmáticas de calcio.

\section{Discusión}

El hiperparatiroidismo durante el embarazo es una alteración poco frecuente en pacientes embarazadas. 


\title{
DESCRIPCIÓN MACROSCOPICA:
}

LÓBULO TIROIDEO DERECHO + PARATIROIDES NFERIOR DERECHA (HEMITIROIDECTOMÍA DERECHA) SE RECIBE EN UN FRASCO CONFORMOL UNA PIEZA PIRIFORME QUE MDIE 5 X 3 X 1 CM, IDENTIFICADA COMO LÓBULO TIROIDEO, AL CORTE SIN ALTERACIONES MACROSCÓPICAS APARENTES, EL PARÉNQUIMA ES HOMOGÉNEO, DE COLOR MARRÓN CLARO, CONSISTENCIA BLANDA, EN LA PERIFERIA DE POLO NFERIOR DE LA PIEZA SE OBSERVA UN FRAGMENTO NODULAR QUE MIDE $0.6 \mathrm{X} 0.5 \mathrm{X}$ 0,5 CM, APARENTEMENTE ENCAPSULADO, DE COLOR GRIS CLARO, SE INCLUYEN CORTES REPRESENTATIVOS. TC. 2 S.1 DR CARRERA/ DRA VIVANCO

\section{LOBULO TIROIDEO DERECHO (HEMTIROIDECTOMIA)}

\author{
CóDIGO: \\ C73X MICROCARCINOMA PAPILAR DE TIROIDES \\ VEAG VERAGREGADO
}

AGREGADO:

MICROCARCINOMA PAPILAR DE TIROIDES INTEGRIDAD DEL ESPECIMEN : NTACTA TAMAÑO DEL ESPECIMEN: 5 X3XI CMS LATERALDAD DE LA NEOPLASLA: DERECHA FOCALDAD: UNIFOCAL TAMAÑO DE LA NEOPLASIA: 0.4X0.3 CMS MEDIDOS EN LAMINLLA TIPO HISTOLOGICO: CARCINOMA PAPILAR EXTENSION EXTRATIROIDEA: LA NEOPLASIA SE ENCUENTRA CONFINADA A LA GLANDULA TIROIDES PERMEASCION VASCULAR: NO IDENTIFICADA INVASION PERINEURAL: NO DENTIFICADA HALLAZGOS ADICIONALES: ADENOMA PARATIROIDEO, TAMAÑO DE LA LESION $0.6 X 0.5 X 0.5$ CMS PT1 PNX

Figura 3. Informe histopatológico de microcarcinoma papilar de tiroides.

Cuando no se diagnostica y no se trata, plantea un riesgo materno y fetal. La operación es la primera opción terapéutica en pacientes con hiperparatiroidismo sintomático. Schnatz, et al. ${ }^{10}$ establecen una serie de indicaciones quirúrgicas en las gestantes con hiperparatiroidismo primario: síntomas de hiperparatiroidismo primario, elevación del calcio sérico $>2.93 \mathrm{mmol} / \mathrm{l}$, antecedentes de crisis de hipercalcemia grave, reducción de la depuración de creatinina en más del $30 \%$ ajustado por edad y estado gestacional sin otra explicación, presencia de nefrolitiasis o nefrocalcinosis, incremento de la excreción urinaria de calcio $>400 \mathrm{mg}$ en orina de 24 horas, afección ósea, escasa colaboración o dificultad en el seguimiento, coexistencia de alguna enfermedad que pueda favorecer la progresión del hiperparatiroidismo o dificultar su control, y petición por parte de la paciente. Los enfoques de mínima invasión han ganado aceptación progresiva como una alternativa segura y eficaz, debido a la utilización de imágenes preoperatorias para sugerir la posición de las glándulas paratiroides. En la actualidad no existen guías para el tratamiento de hiperparatiroidismo durante el embarazo; las opciones disponibles son un enfoque conservador o quirúrgico; la operación durante el segundo trimestre es el tratamiento preferido para los pacientes sintomáticos. Sin embargo, la observación puede ser apropiada en algunas circunstancias (para algunos pacientes con hipercalcemia asintomática y muy leve). En este caso, la paciente contaba con antecedente de nefrolitiasis y pancreatitis grave secundaria a hiperparatiroidismo, un nuevo episodio de pancreatitis grave letal para el binomio. Con base en esta experiencia se sugiere que en una paciente embarazada con pancreatitis y calcio sérico normal o ligeramente elevado se solicite hormona paratiroidea sérica, por la relación que existe entre el hiperparatiroidismo y el desarrollo de pancreatitis. No está bien notificada en las publicaciones médicas la relación que existe entre la hipercalcemia en la paciente embarazada con la presencia de contracciones uterinas y la amenaza de parto de pretérmino. Debe enfatizarse que luego de la realización de la intervención remitieron las contracciones uterinas, por lo que ante una paciente embarazada con HPTP debe realizarse la operación sin importar cuál sea el trimestre en que se encuentre. Si una mujer con diagnóstico de HPTP planea un embarazo, la paratiroidectomía debe practicarse antes de la concepción. Si no se realizó una intervención durante el embarazo debido a una hipercalcemia leve, es importante alertar a los neonatólogos acerca de la posibilidad de hipocalcemia en el recién nacido (debido a la posible supresión de la glándula paratiroidea fetal) ${ }^{4,19}$. En este caso, dado que se operó oportunamente, no se presentaron complicaciones en la madre ni en el feto.

En el diagnóstico diferencial en el segundo y el tercer trimestres se debe tener en cuenta la hipertensión arterial inducida por el embarazo y el síndrome de HELLP (anemia hemolítica, elevación de enzimas hepáticas, trombocitopenia). La mayor parte de los casos de HPTP es esporádica y un gran porcentaje se presenta con un adenoma paratiroideo único. Sin embargo, la posibilidad de un trastorno hereditario siempre debe considerarse en individuos más jóvenes con HPTP; debido a esto, los antecedentes 
familiares son importantes. No debe perderse de vista una posible predisposición genética subyacente, como la neoplasia endocrina múltiple 1 (MEN I), MEN 2, el síndrome del tumor del maxilar, el HPT aislado familiar y la hipercalcemia hipocalciúrica familiar. ${ }^{20-21}$

Otras causas de hipercalcemia a considerar en el embarazo (tirotoxicosis, enfermedad granulomatosa, insuficiencia suprarrenal) se relacionarían de forma típica con una PTH suprimida. La única excepción es la hipercalcemia hipocalciúrica familiar, un trastorno con mutaciones en el gen receptor de detección de calcio. El valor de PTH puede ser normal o moderadamente elevado, pero la excreción urinaria de iones de calcio es en extremo baja. Es necesario excluir la hipercalcemia hipocalciúrica familiar, en la cual los hallazgos son una excreción urinaria de calcio inapropiadamente baja o normal en el contexto de un valor alto de calcio sérico, con PTH alta o normal. El hallazgo de adenomas múltiples debe llevar a solicitar estudios de detección familiar en busca de neoplasias endocrinas múltiples tipos 1 y $2 \mathrm{~A}$, hiperparatiroidismo familiar e hipercalcemia hipocalciúrica familiar ${ }^{22}$.

\section{Conclusión}

El HPTP es una alteración rara en pacientes embarazadas y constituye una causa de morbimortalidad maternofetal evitable. Aunque la experiencia publicada sobre el tema es limitada, se ha planteado que el tratamiento recomendado para una paciente embarazada con hiperparatiroidismo primario es la paratiroidectomía.

\section{Conflicto de intereses}

Los autores declaran que no existe ningún conflicto de intereses.

\section{Responsabilidades éticas}

Protección de personas y animales. Los autores declaran que para esta investigación no se han realizado experimentos en seres humanos ni en animales.
Confidencialidad de los datos. Los autores declaran que han seguido los protocolos de su centro de trabajo sobre la publicación de datos de pacientes.

Derecho a la privacidad y consentimiento informado. Los autores han obtenido el consentimiento informado de los pacientes y/o sujetos referidos en el artículo. Este documento obra en poder del autor de correspondencia.

\section{Bibliografía}

1. Marx SJ. Hyperparathyroid and hypoparathyroid disorders. N Engl J Med. 2000;343:1863-75.

2. Herrera-Martínez AD, Bahamondes-Opazo R, Ortega Palomares, Muñón-Jiménez C, Gálvez-Moreno MA, Quesada Gómez JM. Primary hyperparathyroidism in pregnancy: a two-case report and literature review. Case Rep Obstet Gynecol. 2015;2015:171828. Epub 2015 Mar 29

3. Hirsch D, Kopel V, Nadler V, Levy S, Toledano Y, Tsvetov G. Pregnancy outcomes in women with primary hyperparathyroidism. J Clin Endocrinol Metab, May 2015, 100(5): 2115-2122.

4. McMullen TP, Learoyd DL, Williams DC. Hyperparathyroidism in pregnancy: options for localization and surgical therapy. World J Surg. 2010; 34:1811-16.

5. Ammann P, Irion O, Gust J. Alterations of calcium and phosphate metabolism in primary hyperparathyroidism during pregnancy. Acta Obstet Gynecol Scand. 1993;72:488-92.

6. Kohlmeier L, Marcus R. Calcium disordes of pregnancy. Endocrinol Metab Clin North Am. 1995;24:15-39.

7. Carella MJ, Gossain VV. Hyperparathyroidism and pregnancy. Case report and review. J Gen Intern Med. 1992;7:448-53.

8. Hunter D, Turnbull H. Hyperparathyroidism: generalised osteitis fibrosa with observation upon bones, parathyroid tumor and the normal parathyroid gland. Br J Surg. 1931;19:203-6.

9. Eigelberger MS, Clark $\mathrm{OH}$. Surgical approaches to primary hyperparathyroidism. Endocrinol Metab Clin North Am. 1995;29:479-502.

10. Schnatz PF, Curry SL. Primary hyperparathyroidism in pregnancy: evidence-based management. Obstet Gynecol Surv. 2002; 57:365-76.

11. Pachydakis A, Koutroumanis P, Geyushi B, Hanna L. Primary hyperparathyroidism in pregnancy presenting as intractable hyperemesis complicating psychogenic anorexia: a case report. J Reprod Med. 2008; 53:714.

12. Truong MT, Lalakea ML, Robbins $P$, Friduss M. Primary hyperparathyroidism in pregnancy: a case series and review. Laryngoscope. 2008; 118:1966.

13. Kort KC, Schiller HJ, Numann PJ. Hyperparathyroidism and pregnancy. Am J Surg. 1999;177:66.

14. Chamarthi B, Greene MF, Dluhy RG. Clinical problem-solving. A problem in gestation. N Engl J Med. 2011; 365:843.

15. Kovacs CS, Fulethan G. Calcium and bone disorders during pregnancy and lactation. Endocrinol Metab Clin North Am. 2006;35:21-9.

16. Kelly TR. Primary hyperparathyroidism during pregnancy. Surgery. 1991; 110:1028-34.

17. Davison K, Hawkins DS, Rubin LP. Serum parathyroid hormone in pregnant women determined by an inmunoradiometric assay for intact PTH. J Clin Endocrinol Metab. 1998;67:850-2.

18. Kovacs $\mathrm{C}$, Kronenberg $\mathrm{H}$. Maternal-fetal calcium and bone metabolism during pregnancy, puerperium and lactation. Endocr Rev. 1997;18:837-72.

19. Schnatz PF, Thaxton S. Parathyroidectomy in the third trimester of pregnancy. Obstet Gynecol Surv. 2005; 60:672.

20. Eller-Vainicher C, Chiodini I, Battista C, Viti R, Mascia ML, Massironi S, et al. Sporadic and MEN1-related primary hyperparathyroidism: differences in clinical expression and severity. J Bone Miner Res. 2009;24:1404-10.

21. Callender GG, Rich TA, Perrier ND. Multiple endocrine neoplasia syndromes. Surg Clin North Am. 2008;88:863-895.

22. Rajala MM, Heath H III. Distribution of serum calcium values in patients with familial benign hypercalcemia (hypocalciuric hypercalcemia): evidence for a discrete genetic defect. J Clin Endocrinol Metab. 1987; 65:1039-1041. 